\title{
FLORA BACTERIANA DEL TRACTO DIGESTIVO DE CARACOLES Helix aspersa Müller BAJO DOS SISTEMAS DE CRIANZA
}

\author{
Bacterial Flora in the Digestive Tract of HeliX aspersa Müller Snails under \\ Two Breeding Systems
}

Mirella Villena A. ${ }^{1}$, Siever Morales C., ${ }^{1,2}$, Javier Soto P. ${ }^{3}$, Marco Enciso H. ${ }^{1}$

\section{RESUMEN}

El objetivo del estudio fue comparar la flora bacteriana de intestino y hepatopáncreas del caracol Helix aspersa Müller criados en dos sistemas de producción: intensivo y extensivo. Se colectaron 30 caracoles adultos aparentemente sanos por cada sistema de producción, en seis criaderos ubicados en la provincia de Lima. Se tomó muestras de mucosa intestinal y parénquima del hepatopáncreas de cada individuo, empleando protocolos de aislamientos establecidos para bacterias aerobias y aerobias facultativas. Se aisló bacterias de 15 géneros: Escherichia, Citrobacter, Klebsiella, Enterobacter, Serratia, Hafnia, Proteus, Providencia, Aeromonas, Staphylococcus, Streptococcus, Enterococcus, Micrococcus, Pseudomonas, Acinetobacter; y bacilos gramnegativos no fermentadores (BNF). Se obtuvo 193 cepas en las muestras de caracoles del sistema extensivo, donde el género Escherichia fue el de mayor frecuencia (17.1\%, 33/193); mientras que en caracoles del sistema intensivo se aisló 183 cepas, donde el género Klebsiella fue el de mayor frecuencia (17.5\%, 32/183). Por otro lado, el género más frecuentemente aislado en intestino y hepatopáncreas fue Klebsiella (13.6\%, 25/184 y 17.2\%, 33/192, respectivamente). Bacterias de los géneros Providencia y Micrococcus solo se encontraron en el hepatopáncreas. Hubo diferencias estadísticas en la frecuencia de algunas bacterias aisladas dentro de cada tipo de muestra por efecto del sistema de crianza.

Palabras clave: Helix aspersa, caracol, flora bacteriana, crianza extensiva, crianza intensiva

\section{Abstract}

The objective of this study was to compare the bacterial flora present in the intestine and hepatopancreas of land snails (Helix aspersa Müller) under intensive and extensive breeding systems. Thirty healthy adult snails per production system were collected from six farms located in the province of Lima. Samples from intestinal mucosa and the hepatopancreas were examined from each animal using protocols of microbiological isolation established for aerobic and facultative aerobic bacteria. Bacteria of 15 genuses

\footnotetext{
1. Laboratorio de Microbiología y Parasitología Veterinaria, Facultad de Medicina Veterinaria, Universidad Nacional Mayor de San Marcos, Lima

${ }^{3}$ Laboratorio de Microbiología, Hospital Materno Infantil San Bartolomé, Lima

${ }^{2}$ E-mail:sieverm@gmail.com
} 
were isolated: Escherichia, Citrobacter, Klebsiella, Enterobacter, Serratia, Hafnia, Proteus, Providencia, Aeromonas, Staphylococcus, Streptococcus, Enterococcus, Micrococcus, Pseudomonas, Acinetobacter; and non-fermentative gram-negative bacillus (BNF). It was obtained 193 strains from samples of the extensive production system, where bacteria of the Escherichi genus were predominant (17.1\%, 33/193); and 183 strains were isolated from samples in the intensive production system where the Klebsiella genus was the most frequent (17.5\%, 32/183). Also, the Klebsiella was the most frequent isolated genus in both the intestine and the hepatopancreas (13.6\%, 25/184, and 17.2\%, 33/192 respectively). The Providencia and Micrococcus genuses were only isolated from the hepatopancreas. Statistical differences on the frequency of bacteria isolated between production systems were found.

Key words: Helix aspersa, snail, bacterial flora, extensive breeding, intensive breeding

\section{INTRODUCCIÓN}

Existe una gran demanda a nivel mundial de alimentos para consumo humano de alto contenido proteico y bajos niveles de grasa. Ante esta necesidad, la helicicultura se presenta como una alternativa (Benito, 2004; De la Piedra, 2005; Wallach, 2005). Actualmente, países como España, Italia, Francia, Portugal y Grecia tienen una gran demanda por los caracoles terrestres (Benito, 2004; Wallach, 2005).

El mayor desarrollo en la crianza de caracoles en América Latina lo ostenta Brasil y Argentina. En el Perú, la helicicultura se encuentra en una etapa de incipiente desarrollo y aunque se conocen intentos de productores para afianzar la crianza, no se cumplen los requerimientos del mercado extranjero. La exportación de caracoles se basa mayormente en la recolección de animales silvestres, lo cual contribuye a la depredación de la especie y a la introducción de agentes patógenos que perjudican la productividad de los criaderos (Huertas, 2004; De la Piedra, 2005). La exportación en el Perú se inició en 1998 y hasta el 2006 se ha exportado 144 TM, especialmente a España (SUNAT, 2006).

La principal especie bajo crianza en el país es Helix aspersa Müller, especie prolífica, fitófaga y con alto índice de conversión alimenticia. Sin embargo, el desarrollo de esta industria se encuentra limitado por la presentación de enfermedades bacterianas de origen digestivo (Morales et al., 2006).

Para el diagnóstico adecuado de las enfermedades en $H$. aspersa se requiere conocer la flora bacteriana, debido a que ciertas bacterias pueden comportarse como agentes patógenos oportunistas; así se tiene el caso de la Pseudomona (Watkins y Simkiss, 1990; Fontanillas y García-Cuenca, 2005) y otras potencialmente patógenas que son aisladas de animales aparentemente sanos, tales como Salmonella enterica (Andrews et al., 1975), Enterobacter sp. (Denis et al., 2006), Aeromonas sp. (Kiebre-Toe et al., 2005), Staphylococcus sp. y Vibrio sp. (Watkins y Simkiss, 1990). Incluso, algunos de estos agentes son causantes de enfermedades en humanos y, generalmente, se encuentran asociadas a infecciones nosocomiales e infecciones en pacientes inmunosuprimidos. Sin embargo, se desconoce si estas bacterias se encuentran normalmente en el tracto digestivo, o si su presencia se ve influenciada por algún factor externo.

El objetivo del estudio fue comparar la flora bacteriana mesófila en $H$. aspersa Müller criados en dos sistemas de producción, intensivo y extensivo, como contribución al conocimiento de la microbiología normal de la especie. 


\section{Materiales y Métodos}

Se colectó especímenes de $H$. aspersa de seis criaderos, tres de crianza extensiva y tres de intensiva, ubicados en la provincia de Lima. Las muestras se procesaron en el Laboratorio de Bacteriología y Micología de la Facultad de Medicina Veterinaria, Universidad Nacional Mayor de San Marcos, Lima.

En el sistema de crianza intensivo se emplea el uso de alimento balanceado, así como densidades por área y sistemas de reproducción controlados, entre otras tecnologías, mientras que el tipo de crianza extensiva no se hace uso de estos recursos. Se escogieron al azar 30 caracoles adultos por sistema de crianza, de acuerdo al Teorema del Límite Central de Teoría Avanzada de Probabilidades (Spiegel, 1991). Los caracoles seleccionados se encontraban aparentemente sanos, sin signos clínicos de enfermedad como coloraciones anormales en la cabeza o pie, y decoloración o fragilidad del caparazón.

Los caracoles fueron anestesiados y sacrificados, y se les retiró el caparazón con pinzas. Se expuso el tracto digestivo y, con hisopos estériles, se tomó muestras de la mucosa intestinal y del parénquima del hepatopáncreas. Cada muestra se sembró por agotamiento en un medio de cultivo primario y uno selectivo, Agar Sangre y Agar McConkey, para el aislamiento de aerobios y aerobios facultativos, grampositivos y gramnegativos. Se registró las características de las colonias bacterianas presentes y se realizó la tinción Gram para determinar la morfología bacteriana. Finalmente, se realizaron las pruebas bioquímicas correspondientes para su identificación.

Se determinó la frecuencia de géneros bacterianos del total de las muestras según las variables crianza extensiva e intensiva y órgano muestreado. Para el análisis de la asociación de las variables presencia de bacteria y tipo de crianza se usó la prueba de Chi Cuadrado con el paquete estadístico Stata $9.0^{\circledR}$.

\section{REsultados}

De las 120 muestras procesadas y, de acuerdo a las características bioquímicas, se aislaron 15 géneros de bacterias y bacilos gramnegativos no fermentadores (BNF) no identificados a nivel de género (Cuadro 1). No se aisló cepas de Salmonella.

Se obtuvo 193 cepas en las muestras del sistema extensivo, donde el género Escherichia fue el de mayor frecuencia (17.1\%, 33/193); mientras que en el sistema intensivo se aisló 183 cepas, donde el género Klebsiella fue el de mayor frecuencia (17.5 \%, 32/183). Por otro lado, el género más frecuentemente aislado en intestino como en hepatopáncreas fue Klebsiella (13.6 \%, 25/ 184 y $17.2 \%$, 33/192, respectivamente).

En el hepatopáncreas se aisló bacterias de los 15 géneros, mientras que en el intestino no se encontró los géneros Providencia y Micrococcus. Se encontró diferencias estadísticas en la frecuencia de algunas bacterias aisladas dentro de cada tipo de muestra por efecto del sistema de crianza (Cuadro 1).

\section{Discusión}

De los 15 géneros aislados en el estudio, 10 han sido reportados en caracoles terrestres (H. aspersa), tales como Aeromonas (Kiebre-Toe et al., 2005), Pseudomonas (Watkins y Simkiss, 1990; Fontanillas y García-Cuenca, 2005), Acinetobacter, Staphylococcus, Streptococcus, Micrococcus (Watkins y Simkiss, 1990), Enterococcus (Švec et al., 2001; Charrier et al., 2006), Enterobacter (Watkins y Simkiss, 1990; Charrier et al., 2006; Denis et al., 2006), Klebsiella (Watkins y Simkiss, 1990) y Citrobacter (Charrier et al., 2006). Asimismo, dos géneros han sido reportados en caracoles acuáticos Ampullaria sp.: Escherichia y Proteus (Bartlett y Trust, 1976); 
Cuadro 1. Número de aislamientos de bacterias en muestras de intestino y hepatopáncreas de caracoles $H$. aspersa, según el sistema de crianza ${ }^{1}$

\begin{tabular}{lcccc}
\hline \multirow{2}{*}{ Bacteria } & \multicolumn{2}{c}{ Mucosa intestinal } & \multicolumn{2}{c}{ Parénquima del hepatopáncreas } \\
\cline { 2 - 5 } & S. extensivo & S. intensivo & S. extensivo & S. intensivo \\
\hline Bacilos no fermentadores & $13^{\mathrm{a}}$ & $3^{\mathrm{b}}$ & 16 & 17 \\
Acinetobacter sp. & 1 & 1 & 1 & 1 \\
Aeromonas sp. & $2^{\mathrm{a}}$ & $8^{\mathrm{b}}$ & 6 & 9 \\
Citrobacter sp. & 11 & 13 & 10 & 10 \\
Enterobacter sp. & 13 & 10 & 10 & 11 \\
Enterococcus sp. & 2 & 4 & 1 & 4 \\
Escherichia sp. & 13 & 12 & $20^{\mathrm{a}}$ & $13^{\mathrm{b}}$ \\
Hafnia sp. & 2 & 3 & 4 & 3 \\
Klebsiella sp. & $8^{\mathrm{a}}$ & $16^{\mathrm{b}}$ & $9^{\mathrm{a}}$ & $16^{\mathrm{b}}$ \\
Micrococcus sp. & -- & -- & 1 & 1 \\
Proteus sp. & 4 & 2 & $8^{\mathrm{a}}$ & $0^{\mathrm{b}}$ \\
Providencia sp. & -- & -- & 1 & 1 \\
Pseudomonas sp. & 2 & 2 & 2 & 1 \\
Serratia sp. & 12 & 11 & 10 & 8 \\
Staphylococcus sp. & 4 & 4 & 3 & 3 \\
Streptococcus sp. & 5 & 3 & 5 & 2 \\
\hline 1 30 caracoles por sistemas de & producción y una muestra de mucosa intestinal y otra del \\
a,bepatopáncreas por individuo & Superíndices diferentes dentro de cada tipo de bacteria y tipo de muestra indican diferencia \\
significativa (p<0.05) & & & &
\end{tabular}

y los tres géneros restantes, Serratia, Hafnia, y Providencia, se reportan por primera vez. Por otro lado, los géneros Escherichia y Proteus, como algunos BNF, han sido aislados por primera vez en caracoles $H$. aspersa Müller.

La bacteria más frecuente en muestras de hepatopáncreas en el sistema de crianza intensiva fue del género Klebsiella, en tanto que en el sistema extensivo fueron Escherichia y Proteus, lo cual indica que existen factores que no están siendo adecuadamente controlados en el galpón, como la contaminación del alimento, el agua, la insuficiente higiene en la infraestructura o el deficiente aseo del personal en el primer caso, así como algún tipo de contaminación del medio ambiente en el segundo caso.

Entre otras bacterias importantes para la crianza comercial del $H$. aspersa se encuentran las Aeromonas (Kiebre-Toe et al., 2005) y Pseudomonas (Watkins y Simkiss, 1990; Fontanillas y García-Cuenca, 2005), descritas en caracoles enfermos donde se desencadenó una elevada mortalidad, y por lo tanto, deben considerarse como importantes desde el punto de vista sanitario. Sin embargo, la frecuencia de aislamiento de Aeromonas en el estudio de Kiebre-Toe et al. (2005) fue mayor al del presente estudio, y donde las muestras se tomaron de caracoles sin signos clínico de enfermedad. Por otro 
lado, es posible que el tipo de crianza haya influido en el aislamiento de Aeromonas ya que aquí se encontró una mayor frecuencia en caracoles bajo crianza intensiva.

Kiebre-Toe et al. (2005) sostienen que cepas del grupo Aeromonas hydrophila se encuentran asociadas a una enfermedad de verano producida en caracoles. El hallazgo en este estudio de cepas de Aeromonas en caracoles sanos sugiere que probablemente las cepas aisladas no sean del grupo $A$. hydrophila. Además, Watkins y Simkiss (1990) reportan la presencia de Pseudomonas sp. en caracoles sanos.

Entre las bacterias de mayor importancia en salud pública, por ser una la principales causantes de gastroenteritis, se encuentra la Salmonella sp. (Jenkins y Gillespie, 2006), razón por la que se utilizó un protocolo específico de aislamiento desarrollado por el Instituto Nacional de Salud (INS, 2001). Sin embargo, ningún caracol fue positivo a esta bacteria. Otros estudios lograros aislar Salmonella enterica de 13 diferentes serotipos en muestras de tracto gastrointestinal en el 31\% de la población (Andrews et al., 1975), incluyendo en caracoles enfermos en el Perú (Morales et al., 2006); lo que sugiere el potencial riesgo para la salud pública que representa el consumo de caracoles.

Los caracoles $H$. aspersa son utilizados para el consumo humano, de modo que la aplicación de buenas prácticas en la producción, procesamiento y manufactura resulta de mucha importancia, ya que la mayoría de bacterias aisladas en el presente estudio se comportan como patógenos oportunistas, constituyendo un peligro potencial para la salud pública.

\section{Conclusiones}

- La flora bacteriana presente en caracoles (H. aspersa Müller) criados en sistemas de producción intensiva y extensiva corresponden a bacterias mesófilas potencialmente patógenas.
- Se encontró diferencias estadísticas entre la flora proveniente de crianza extensiva donde predominan los géneros Klebsiella, Aeromonas y bacilos no fermentadores y los de crianza intensiva donde predominan los géneros Escherichia, Klebsiella y Proteus.

\section{Agradecimientos}

Los autores expresan su agradecimiento a los profesores Felipe San Martín H. y Sonia Calle E. por su valioso aporte en la redacción del presente documento.

\section{Literatura Citada}

1. Andrews WH, Wilson CR, Romero A, Poelma PL. 1975. The Moroccan food snail, Helix aspersa, as a source of Salmonella. Appl Microbiol 29: 328-330.

2. Bartlett K, Trust T. 1976. Isolation of Salmonellae and other potential pathogens from the freshwater aquarium snail Ampullaria. Appl. Env Microbiol 31: 635-639.

3. Benito M. 2004. Evaluación técnica económica de una crianza intensiva de Caracoles (Helix aspersa). Tesis de Ingeniero Agrónomo. Santiago de Chile: Pontificia Universidad Católica. 62 p.

4. Charrier M, Fonty G, GaillardMartinie B, Ainouche K, Andant G. 2006. Isolation and characterization of cultivable fermentative bacteria from the intestine of two edible snails, Helix pomatia and Cornu aspersum (Gastropoda: Pulmonata). Biol Res 39: 669-681.

5. De la Piedra R. 2005. Biología del caracol (Helix aspersa Muller) y propuesta de instalación de un criadero mixto modificado. Tesina de Médico Veterinario. Lima: Universidad Nacional Mayor de San Marcos. 88 p.

6. Denis C, Cadot P, Leguerinel I, Thuault D, Sohier D. 2006. Heat resistance of coliform species isolated from cooked ham, snail flesh, and 
'bouche'es a'la reine'. Lett Appl Microbiol 42: 160-164.

7. Fontanillas J, García-Cuenca I. 2005. El caracol y la helicicultura. $2^{\text {a }}$ ed. España: Mundi-Prensa. 142 p.

8. Huertas W. 2004. Evaluación económica para la instalación de una granja de crianza y reproducción de caracoles de tierra (Helix aspersa) con fines de exportación. Lima: Universidad Nacional Agraria La Molina. 75 p.

9. [INS] Instituto Nacional de Salud. 2001. Guía de procedimientos: Actualización del diagnóstico de enterobacterias y sensibilidad antibiótica. Laboratorio de Referencia Nacional e Enteropatógenos CNLSP. Lima: Ministerio de Salud. $46 \mathrm{p}$.

10. Jenkins C, Gillespie S. 2006. Salmonella spp. En: Gillespie S, Hawkey $\mathrm{P}$ (eds). Principles and practice of clinical bacteriology. Cap 29. $2^{\text {a }}$ ed. UK: John Wiley. p 367-376.

11. Kiebre-Toe MB, Lancheretz A, Villard L, Richard Y, Kodjo A. 2005. Pulsedfield gel electrophoresis profiles of aeromonads isolated from healthy and diseased Helix aspersa from French snail farms. Can J Microbiol 51: 817-820.
12. Morales S, Calle S, Pinto C. 2006. Salmonella enterica en caracoles (Helix aspersa Muller) en sistemas de crianza intensiva. En: XXIX Reunión Científica Anual de la Asociación Peruana de Producción Animal. Huancayo: APPA. p 530-531.

13. Spiegel M. 1991. Estadística. $2^{\mathrm{a}}$ ed. Mexico DF: McGraw Hill. p 186-188.

14. SUNAT. 2006. Lima: DATATRADE. [Internet], [13 febrero 2007]. Disponible en: http://www.datatrade.com.pe

15. Švec P, Devriese L, Sedláèek I, Baele $M$, Vancanneyt M, Haesebrouck F, Swings J, Doškaø J. 2001. Characterization of yellow-pigmented and motile enterococci isolated from intestines of the garden snail Helix aspersa. J Appl Microbiol 92: 951-957.

16. Wallach R. 2005. Helicicultura: Cría de caracoles terrestres. Tesis de Ingeniero Agrónomo. Chile: Pontificia Universidad Católica. $71 \mathrm{p}$.

17. Watkins B, Simkiss K. 1990. Interactions between soil bacteria and the Molluscan alimentary tract. J Moll Stud 56: 267-274. 